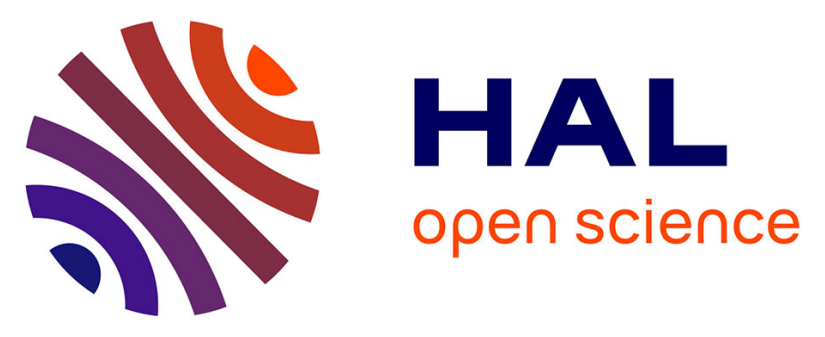

\title{
Utilisation de polynômes de Tchebychev pour l'identification de modèles à temps continu de robots
} Minh Tu Pham, Corinne Rouby, Jérémy Lizandier, Didier Rémond, Alexandre Janot, Pierre Olivier Vandanjon, Maxime Gautier

\section{- To cite this version:}

Minh Tu Pham, Corinne Rouby, Jérémy Lizandier, Didier Rémond, Alexandre Janot, et al.. Utilisation de polynômes de Tchebychev pour l'identification de modèles à temps continu de robots. Journal Européen des Systèmes Automatisés (JESA), 2012, 46 (6/7), pp.779-798. 10.3166/jesa.46.779-798 . hal-00782079

\section{HAL Id: hal-00782079 https://hal.science/hal-00782079}

Submitted on 1 Jun 2019

HAL is a multi-disciplinary open access archive for the deposit and dissemination of scientific research documents, whether they are published or not. The documents may come from teaching and research institutions in France or abroad, or from public or private research centers.

$$
\text { Copyright }
$$

L'archive ouverte pluridisciplinaire $\mathbf{H A L}$, est destinée au dépôt et à la diffusion de documents scientifiques de niveau recherche, publiés ou non, émanant des établissements d'enseignement et de recherche français ou étrangers, des laboratoires publics ou privés. 


\title{
Utilisation de Polynômes de Tchebychev pour l'Identification de Modèles à Temps Continu de Robots
}

\author{
Minh Tu PhaM ${ }^{1}$ - Corinne RoubY ${ }^{2}$ - Jérémy LizandieR ${ }^{1}$ - Di- \\ dier RÉMOND ${ }^{3}$ - Alexandre JANOT $^{4}$ - Pierre-Olivier VANDAN- \\ JON $^{5}$ - Maxime GAUTIER ${ }^{6}$ \\ 1 Laboratoire Ampère, UMR CNRS 5005, \\ Université de Lyon, INSA-Lyon, F-69621 Villeurbanne Cedex, France \\ (minh-tu.pham, jeremy.lizandier)@insa-lyon.fr \\ 2 Unité de Mécanique, \\ ENSTA ParisTech, Chemin de le Hunière, 91761 Palaiseau Cedex, France \\ corinne.rouby@ensta-paristech.fr \\ 3 LaMCoS, CNRS UMR5259, \\ Université de Lyon, INSA-Lyon, F-69621 Villeurbanne Cedex, France \\ didier.remond@insa-lyon.fr \\ 4 ONERA DCSD, \\ 2 Avenue Edouard Belin - BP 74025, 31055 Toulouse Cedex 04, France \\ Alexandre.Janot@onera.fr \\ 5 Laboratoire Central des Ponts et Chaussées, \\ 44341 Bouguenais Cedex, France \\ pierre-olivier.vandanjon@lcpc.fr \\ 6 IRCCyN, UMR CNRS 6597, \\ Université de Nantes, 44321 Nantes Cedex 03, France \\ maxime.gautier@irccyn.ec-nantes.fr
}

RÉSUMÉ. Dans cet article, une présentation des propriétés des polynômes de Tchebychev et une formulation dérivée utilisant ces polynômes sont effectuées. La méthode d'identification présentée consiste à appliquer une transformation linéaire sur le système d'équations qui régit le processus. Pour cela, l'opérateur mathématique utilisé s'appuie sur une décomposition du 


\section{2e soumission à $J E S A$}

signal dans une base formée de polynômes orthogonaux. Nous montrons que cette projection se comporte comme un filtre passe-bande, de telle sorte qu'une seule opération est nécessaire pour le pré-traitement des données avant le processus d'identification, à savoir choisir la dimension de la base des polynômes Tchebychev. Une identification expérimentale en boucle fermée d'un robot à 2 axes avec cette méthode est effectuée dans une dernière partie. Les résultats obtenus sont comparés et analysés avec d'autres techniques utilisées en robotique.

ABSTRACT. In this paper, a presentation of the properties of Tchebychev polynomials and a formulation derived using these polynomials are performed. The identification method presented consists in applying a linear transformation to the set of equations of the process. For this, the mathematical operator used is based on a decomposition of the signal in a basis of orthogonal polynomials. We show that this projection acts as a bandpass filter, so that only one operation is required for the data preprocessing before the identification, namely the choice of the Chebyshev polynomials basis size. An experimental closed-loop identification of a 2-axis robot with this method is performed in a final section. The results are compared and analyzed with other techniques used in robotics.

MOTS-CLÉS : polynômes orthogonaux, analyse fréquentielle, identification en boucle fermée, problème inverse, robotique

KEYWORDS: orthogonal polynomials, frequency analysis, closed-loop identification, inverse problem, robotics 


\section{Introduction}

Dans le contexte de la mécanique, la prédiction du comportement dynamique de mécanismes ou de structures complexes exige des modèles fins. Malheureusement, les conditions aux frontières, le caractère incertain des paramètres et les coefficients de viscosité rendent l'identification de tels modèles délicate. L'une des difficultés principales se situe dans la transformation des équations différentielles de ce type de procédé en un système d'équations algébriques. Traditionnellement, lorsque les hypothèses de linéarité sont possibles et vérifiées, une forme discrète des équations à temps continu est obtenue en appliquant la transformée en $\mathrm{Z}$. Ceci conduit à des équations récurrentes équivalentes quand les signaux sont récoltés à pas constant avec un échantillonneur et un bloqueur d'ordre zéro. À ce stade, le choix de la période d'échantillonnage peut être critique car un suréchantillonnage rend difficile l'identification des paramètres physiques (Mensler, 1999). Au cours de ces dernières décennies, des méthodes alternatives ont été proposées et référencées sous la terminologie méthodes d'identification à temps continu (Young, 1981; Mensler, 1999; Ahmed et al., 2006; Chou et al., 1999; Larsson and Soderstrom, 2002). Parmi ces approches, des méthodes s'appuyant sur des fonctions orthogonales ont été employées à travers une formulation intégrale des équations différentielles. Leur principal avantage est qu'elles transforment l'intégration directe des signaux en intégration plus simple de ces fonctions en se servant d'une matrice carrée qui dépend des fonctions orthogonales. Par conséquent, les équations régissant le comportement du système mécanique peuvent être transformées en un jeu d'équations algébriques. Un autre avantage de ces fonctions est leur propriété d'orthogonalité qui permet de décomposer un signal sur une base polynomiale. Dans (Pacheco and Steffen, 2002), les auteurs comparent différentes bases orthogonales telles que les polynômes de Jacobi (Horng and Chou, 1986), d'Hermite (Paraskevopoulos and Kekkeri, 1983), de Legendre (Paraskevopoulos and Mouroutsos, 1985) ou de Tchebychev (Mohan and Datta, 1988), les fonctions Walsh (Karanam et al., 1978) et, naturellement, les séries de Fourier (Mohan and Datta, 1989). Ils y mentionnent également la facilité de mise en oeuvre de la formulation intégrale dans le contexte de l'identification de problèmes inverses et la simplification du calcul dans des problèmes d'analyse de sensibilité. Dans (Pacheco and Steffen, 2004), les auteurs emploient également cette formulation intégrale en présence des nonlinéarités telles que l'oscillateur de Duffing ou les frottements secs. Dans le domaine de l'identification du comportement non linéaire, d'autres auteurs ont proposé des approches semblables utilisant des polynômes de Tchebychev (Masri and Caughey, 1979) ou employant d'autres polynômes ou séries chronologiques (Chen and Tomlinson, 1996; Horng and Chou, 1987). Dans (Ghanem and Romeo, 2000; Ghanem and Romeo, 2001), les auteurs présentent des travaux reposant sur une formulation dérivée à partir d'ondelettes. Les auteurs arrivent aux mêmes conclusions sur la pertinence de l'utilisation des fonctions orthogonales pour résoudre des problèmes d'identification dynamique de systèmes à comportement linéaire ou non linéaire à paramètres variants ou non. Il apparaît à travers cette bibliographie que l'approximation des opérateurs de dérivation est beaucoup plus commode pour l'identification, lorsque l'évaluation des états initiaux n'est pas exigée, et pour des problèmes inverses, où au- 
cune inversion matricielle n'est nécessaire. Finalement Pacheco et Steffen montrent dans (Pacheco and Steffen, 2004) que les résultats d'identification obtenus avec des polynômes de Tchebychev, de Jacobi ou de Legendre ne sont pas satisfaisants quand des excitations aléatoires sont appliquées, bien qu'aucune explication n'ait été fournie. Cette difficulté s'explique par la forme des polynômes de Tchebychev qui aboutit à des projections de faible qualité pour des signaux à large bande de fréquences. Par conséquent, une attention particulière doit être prêtée à la qualité de la projection des signaux avant l'étape d'estimation paramétrique. Cet inconvénient des polynômes de Tchebychev nous oblige à proposer une méthodologie plus générale d'identification qui peut être facilement prolongée à d'autres polynômes classiques.

L'organisation de l'article est la suivante : Dans un premier temps les propriétés des polynômes de Tchebychev et une formulation dérivée utilisant ces polynômes sont rappelées. La méthode d'identification présentée dans ce article consiste à appliquer une transformation linéaire sur le système d'équations qui régit le processus. Pour cela, l'opérateur mathématique utilisé s'appuie sur une décomposition du signal dans une base formée de polynômes orthogonaux. Nous montrons dans un second temps que cette projection a l'avantageuse propriété de se comporter comme un filtre passebande, de telle sorte qu'une seule opération est nécessaire pour le pré-traitement des données avant le processus d'identification. L'identification en boucle fermée d'un robot à 2 axes avec cette méthode est effectué dans une dernière partie. Les résultats obtenus sont comparés et analysés avec d'autres techniques utilisées en robotique.

\section{Polynômes Orthogonaux}

\subsection{Base de polynômes orthogonaux}

L'approximation des fonctions à valeurs réelles est un problème réputé difficile en analyse numérique. Mis à part les cas académiques, les solutions d'équations différentielles ou aux dérivées partielles ne peuvent s'obtenir par simples quadratures et les solutions recherchées s'écrivent sous forme d'un développement en série. Le développement en série de Taylor peut être par exemple une réponse à ces problèmes mais la convergence peut s'avérer lente ou son emploi inadapté. Dès lors qu'on a défini une norme de convergence uniforme, il est possible de déterminer une fonction polynomiale $P$ appelée polynôme de meilleure approximation d'une fonction $x$ pour la norme considérée. L'existence de ce polynôme est assurée par le théorème de StoneWeierstrass. L'étude présentée dans ce article donne suite à une méthode originale (Rémond et al., 2008) visant à exploiter les propriétés de dérivation des familles de polynômes orthogonaux. La famille considérée est la base de Tchebychev type I mais la technique utilisée est tout aussi bien applicable aux familles de Tchebychev II ou encore Legendre. Le $i$-ème terme de la suite polynomiale de Tchebychev définie sur l'intervalle de temps $I=[-1 ; 1]$ s'écrit :

$$
P_{i}(\tau)=\cos (i \arccos (\tau)) \quad i \in\{1,2, \ldots, n\}
$$


L'ensemble $\left\{P_{0}, P_{1}, \ldots, P_{n}\right\}$ forme une base orthogonale de l'ensemble $E$ des polynômes de degré inférieur ou égal à $n$ par rapport au produit scalaire défini par :

$$
<P, Q>=\int_{-1}^{1} \frac{P(\tau) Q(\tau)}{\sqrt{1-\tau^{2}}} d \tau
$$

où $P$ et $Q$ sont deux éléments de $E$.

\subsection{Développement d'une fonction continue sur la base de Tchebychev}

Soit la fonction $x: t \mapsto x(t)$ continue sur l'intervalle $\left[t_{0} ; t_{f}\right]$ et la fonction $x_{I}: \tau \mapsto x_{I}(\tau)$ correspondant à $x$ sur l'intervalle $I=[-1 ; 1]$ et définie par

$$
x_{I}(\tau(t))=x(t)
$$

où

$$
\tau(t)=\frac{2 t-t_{f}-t_{0}}{t_{f}-t_{0}}
$$

On montre que $x$ peut alors se décomposer de telle sorte que :

$$
x(t)=\sum_{i=0}^{\infty} \tilde{x}_{i} P_{i}(\tau(t))
$$

Afin de reconstituer de manière exacte la fonction $x$, nous devons disposer d'une base formée par une infinité de polynômes. Dans la pratique, une troncature à l'ordre $n$ est nécessaire, menant à une approximation, notée $T_{n}[x]$, de la fonction $x$, soit :

$$
T_{n}[x](t)=\sum_{i=0}^{n} \tilde{x}_{i} P_{i}(\tau(t))
$$

ou encore sous forme vectorielle :

$$
T_{n}[x](t)=\left[\begin{array}{lllll}
\tilde{x}_{0} & \tilde{x}_{1} & \ldots & \tilde{x}_{n-1} & \tilde{x}_{n}
\end{array}\right]\left\{P^{n}(\tau(t))\right\}
$$

avec

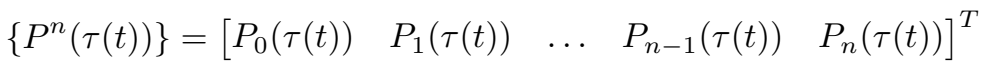

Ces dernières composantes $\tilde{x}_{i}$ peuvent s'obtenir analytiquement en projetant le signal $x$ sur la base orthogonale $\left\{P_{0}, P_{1}, \ldots, P_{n}\right\}$ :

$$
\tilde{x}_{i}=\frac{\left\langle x_{I}, P_{i}\right\rangle}{\left.<P_{i}, P_{i}\right\rangle}
$$

L'échantillonnage de l'équation [6] le long d'une trajectoire à différents instants $t_{i} \in\left\{t_{0}, \ldots, t_{f}\right\}$ permet d'écrire la relation suivante :

$$
\left[T_{n}[x]\right]=[\tilde{x}][P]
$$


avec

$$
\begin{aligned}
& {\left[T_{n}[x]\right]=\left[\begin{array}{lllll}
T_{n}[x]\left(t_{0}\right) & T_{n}[x]\left(t_{1}\right) & \ldots & T_{n}[x]\left(t_{f-1}\right) & T_{n}[x]\left(t_{f}\right)
\end{array}\right]} \\
& {[\tilde{x}]=\left[\begin{array}{lllll}
\tilde{x}_{0} & \tilde{x}_{1} & \ldots & \tilde{x}_{n-1} & \tilde{x}_{n}
\end{array}\right]} \\
& {[P]=\left[\begin{array}{ccc}
P_{0}\left(\tau\left(t_{0}\right)\right) & \ldots & P_{0}\left(\tau\left(t_{f}\right)\right) \\
P_{1}\left(\tau\left(t_{0}\right)\right) & \ldots & P_{1}\left(\tau\left(t_{f}\right)\right) \\
\vdots & \ddots & \vdots \\
P_{n-1}\left(\tau\left(t_{0}\right)\right) & \ldots & P_{n-1}\left(\tau\left(t_{f}\right)\right) \\
P_{n}\left(\tau\left(t_{0}\right)\right) & \ldots & P_{n}\left(\tau\left(t_{f}\right)\right)
\end{array}\right]}
\end{aligned}
$$

De cette manière il est possible d'estimer les composantes $\tilde{x}_{i}$ par une méthode de moindres carrés menant à l'expression :

$$
[\tilde{x}]=\left[T_{n}[x]\right][P]^{+}
$$

où $[P]^{+}$est la matrice pseudo-inverse de $[P]$. Pour un nombre approprié de polynômes, on obtient alors les mêmes valeurs de coefficients qu'à l'aide de l'expression [9], dont le calcul est donné dans l'annexe 5.1.

\subsection{Estimation des dérivées successives}

Parmi les propriétés des polynômes, notons que la dérivée de chaque polynôme $P_{i}$ se décompose en somme de polynômes de degrés inférieurs. L'expression obtenue est décrite ci-dessous pour le cas de la famille de Tchebychev type I (Rémond et al., 2008) :

$$
\frac{d P_{n}}{d t}= \begin{cases}P_{0} & \text { pour } n=1 \\ 2 n \sum_{m=0}^{n / 2-1} P_{2 m+1} & \text { pour } n \geq 2 \text { et } n \text { pair } \\ n P_{0}+2 n \sum_{m=1}^{(n-1) / 2} P_{2 m} & \text { pour } n \geq 3 \text { et } n \text { impair }\end{cases}
$$

Il est ainsi possible d'introduire une matrice carrée $D$, dont l'expression est donnée dans l'annexe 5.2, telle que :

$$
\begin{aligned}
T_{n}[\dot{x}](t) & =\frac{2}{t_{f}-t_{0}}\left[\begin{array}{lllll}
\tilde{x}_{0} & \tilde{x}_{1} & \ldots & \tilde{x}_{n-1} & \tilde{x}_{n}
\end{array}\right]\left\{\dot{P}_{n}(\tau(t))\right\} \\
& =\frac{2}{t_{f}-t_{0}}\left[\begin{array}{lllll}
\tilde{x}_{0} & \tilde{x}_{1} & \ldots & \tilde{x}_{n-1} & \tilde{x}_{n}
\end{array}\right] D\left\{P_{n}(\tau(t))\right\}
\end{aligned}
$$


Et de la même manière, pour la dérivée seconde :

$$
\begin{aligned}
T_{n}[\ddot{x}](t) & =\left(\frac{2}{t_{f}-t_{0}}\right)^{2}\left[\begin{array}{lllll}
\tilde{x}_{0} & \tilde{x}_{1} & \ldots & \tilde{x}_{n-1} & \tilde{x}_{n}
\end{array}\right]\left\{\ddot{P}_{n}(\tau(t))\right\} \\
& =\left(\frac{2}{t_{f}-t_{0}}\right)^{2}\left[\begin{array}{lllll}
\tilde{x}_{0} & \tilde{x}_{1} & \ldots & \tilde{x}_{n-1} & \tilde{x}_{n}
\end{array}\right] D^{2}\left\{P_{n}(\tau(t))\right\}
\end{aligned}
$$

\subsection{Analyse fréquentielle de la projection sur la base de Tchebychev}

La dimension de la base de projection (i.e. le degré maximum du plus grand polynôme de la base) influence la qualité de l'approximation [6] du signal. Afin de caractériser le comportement d'un tel opérateur, une étude portant sur l'influence du contenu spectral observé par rapport au degré maximum de polynômes choisis est présentée ci-dessous. Dans un premier temps, l'analyse est menée sur un signal sinusoïdal de fréquence $f: x_{f}=\sin (2 \pi f t)$. Un nombre de 15 points par période a été défini arbitrairement pour réaliser une telle étude. L'atténuation en amplitude en décibel entre le signal original et le signal reconstitué $T_{n}\left[x_{f}\right]$ après projection est représentée par la nappe de la figure 1.

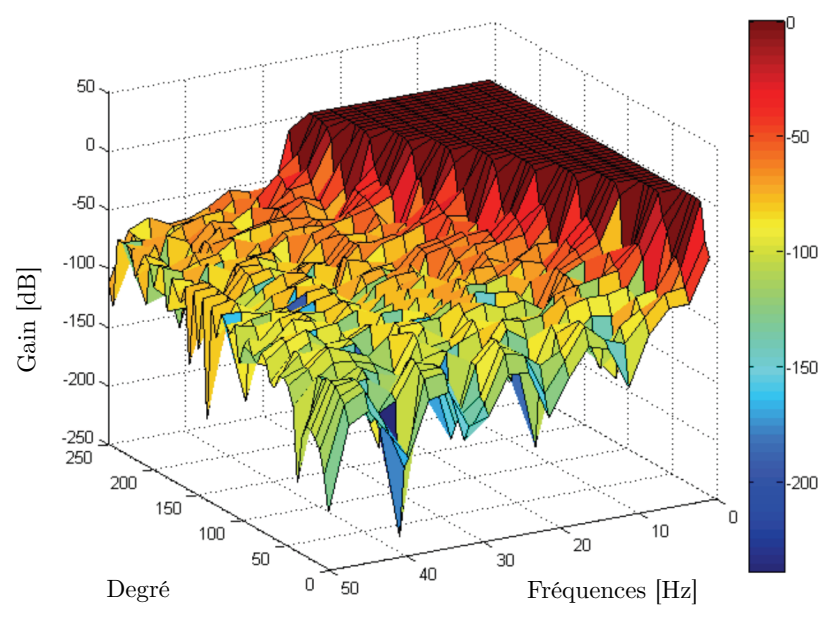

Figure 1. Atténuation du signal reconstitué par rapport au signal initial, définie par $20 \log A(n, f)$ où $A(n, f)$ est le rapport des amplitudes de $T_{n}\left[x_{f}\right]$ sur $x_{f}$ 
Cette courbe montre que lorsque la fréquence $f$ croît, une augmentation de la dimension de la base de projection est nécessaire pour reconstituer correctement le signal original. Ainsi l'opérateur linéaire de projection polynomiale peut être considéré comme un filtre passe bas avec la dimension de la base comme paramètre variant en fonction de la fréquence de coupure du filtre.

Un autre aspect que nous avons souhaité analyser est le comportement fréquentiel de l'opérateur de dérivation construit sur la base de Tchebychev en fonction de la fréquence normalisée $f_{N}$. Cette dernière est définie par l'équation suivante :

$$
f_{N}=\frac{f}{f_{e}}
$$

où $f_{e}$ est la fréquence d'échantillonnage.

On constate dans la pratique que si le signal $T_{n}[\dot{x}]$ approximant la dérivée parfaite $\dot{x}$ est en phase avec celle-ci, en revanche ce signal reconstruit subit une distorsion en amplitude en fonction de la fréquence du signal $x$. Sur la figure 2 sont représentées l'atténuation en amplitude de la dérivée exacte $\dot{x}_{f}$ du signal par rapport au signal initial $x_{f}$ et l'atténuation en amplitude de la dérivée reconstruite $T_{n}\left[\dot{x}_{f}\right]$ par rapport au signal initial $x_{f}$, en fonction de la fréquence du signal sinusoïdal et pour différentes dimensions de la base de projection. L'analyse des figures 2 et 3 montre clairement que l'opérateur de dérivation par projection polynomial se comporte comme un filtre. Plus le degré de décomposition augmente et plus haute est la fréquence de coupure. Il devient alors possible de choisir la dimension de la base de projection en fonction de la fréquence de coupure désirée. L'opérateur peut alors être assimilé à un filtre passe bande, composé d'un filtre dérivateur en basses fréquences et d'un filtre passe bas en haute fréquence.

De manière similaire les figures 4 et 5 montrent que le comportement fréquentiel de l'opérateur de dérivation polynomial pour l'estimation de la dérivée seconde $T_{n}\left[\ddot{x}_{f}\right]$ est celui d'un filtre passe bande.

\section{Application à l'identification d'un robot à deux degrés de libertés}

\subsection{Modèle d'identification}

La démarche d'identification généralement adoptée en robotique consiste à utiliser le modèle dynamique inverse du robot qui est linéaire par rapport aux paramètres dynamiques à estimer. Le modèle dynamique inverse d'un tel robot exprime le vecteur de couples moteur $\Gamma$ (l'entrée de commande) en fonction des coordonnées généralisées (le vecteur d'état et sa dérivée). Il peut être obtenu à partir du lagrangien ou de l'algorithme de Newton Euler. Il s'écrit sous la forme suivante (Khalil and Dombre, 1999; Kozlowski, 1998; Sciavicco and Siciliano, 2000) :

$$
\Gamma=M\left(q, \theta_{S}\right) \ddot{q}+H\left(q, \dot{q}, \theta_{S}\right)
$$



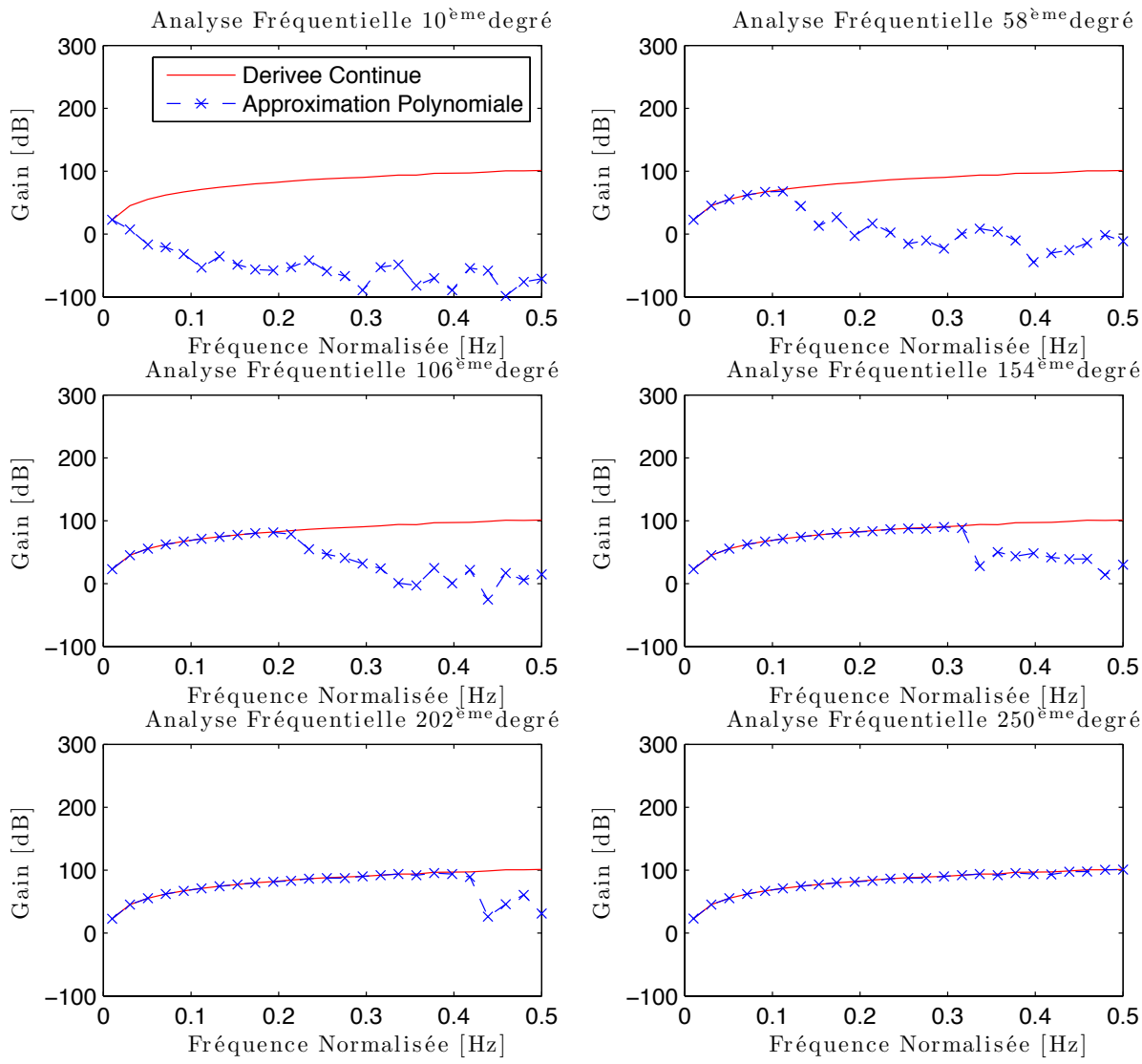

Figure 2. Caractérisation fréquentielle de l'opérateur dérivé par projection polynomiale. Fréquence d'échantillonnage de $100 \mathrm{~Hz}$

$q=\left[\begin{array}{lll}q_{1} & \cdots & q_{N}\end{array}\right]^{T}$ désigne le vecteur des $N$ positions des articulaires du robot. $\dot{q}=\left[\begin{array}{lll}\dot{q}_{1} & \cdots & \dot{q}_{N}\end{array}\right]^{T}, \ddot{q}=\left[\begin{array}{lll}\ddot{q}_{1} & \cdots & \ddot{q}_{N}\end{array}\right]^{T}$ et $\Gamma=\left[\begin{array}{lll}\Gamma_{1} & \cdots & \Gamma_{N}\end{array}\right]^{T}$ correspondent respectivement aux vecteurs des vitesses articulaires, des accélérations articulaires et des couples moteurs. $M\left(q, \theta_{S}\right)$ et $H\left(q, \dot{q}, \theta_{S}\right)$ désignent respectivement la matrice d'inertie et le vecteur des forces centrifuges, de Coriolis, de gravité et de frottements du robot.

L'équation [16] peut s'écrire sous une forme linéaire par rapport aux paramètres dynamiques $\theta_{S}$ (Mayeda et al., 1990; Gautier, 1990; Gautier and Khalil, 1990) :

$$
\Gamma=D_{S}(q, \dot{q}, \ddot{q}) \theta_{S}
$$




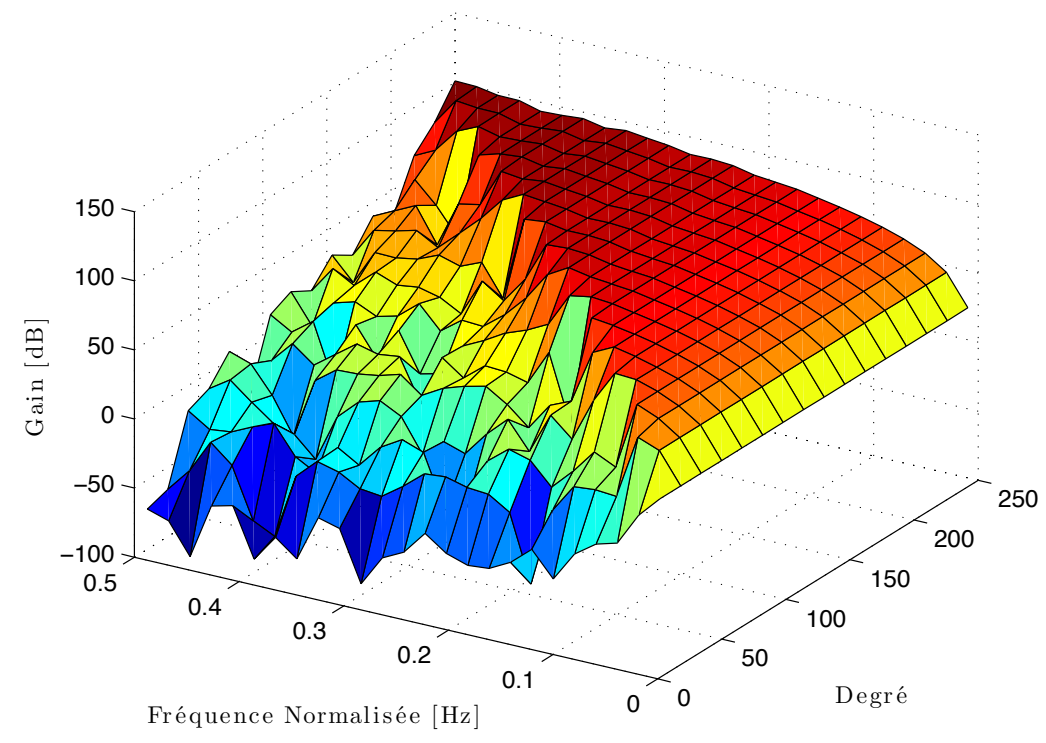

Figure 3. Distorsion en amplitude du filtre dérivateur en fonction de la fréquence du signal d'entrée et du degré des polynômes

Le système étudié est un robot planaire SCARA à deux articulations rotoïdes (figure 6) sans effet de gravité et développé par le laboratoire IRCCyN à Nantes. Soit $q=\left[q_{1}, q_{2}\right]^{T}$ le vecteur des variables articulaires. La matrice de régression $D_{S}$ du modèle dynamique d'identification [17] s'écrit :

$$
D_{s}(q, \dot{q}, \ddot{q})=\left(\begin{array}{cc}
\ddot{q}_{1} & 0 \\
\dot{q}_{1} & 0 \\
\operatorname{sign}\left(\dot{q}_{1}\right) & 0 \\
\ddot{q}_{1}+\ddot{q}_{2} & \ddot{q}_{1}+\ddot{q}_{2} \\
\left(2 \ddot{q}_{1}+\ddot{q}_{2}\right) C_{2}-\dot{q}_{2}\left(2 \dot{q}_{1}+\dot{q}_{2}\right) S_{2} & \ddot{q}_{1} C_{2}+\dot{q}_{1}^{2} S_{2} \\
-\left(2 \ddot{q}_{1}+\ddot{q}_{2}\right) S_{2}-\dot{q}_{2}\left(2 \dot{q}_{1}+\dot{q}_{2}\right) C_{2} & \dot{q}_{1}^{2} C_{2}-\ddot{q}_{1} S_{2} \\
0 & \dot{q}_{2} \\
0 & \operatorname{sign}\left(\dot{q}_{2}\right)
\end{array}\right)^{T}
$$

avec $C_{2}=\cos \left(q_{2}\right)$ et $S_{2}=\sin \left(q_{2}\right)$; et le vecteur des paramètres inconnus est défini par:

$$
\theta_{S}=\left[\begin{array}{llllllll}
Z Z R_{1} & F_{v 1} & F_{s 1} & Z Z_{2} & L M X_{2} & L M Y_{2} & F_{v 2} & F_{s 2}
\end{array}\right]^{T}
$$

avec $Z Z R_{1}=Z Z_{1}+M_{2} L^{2}, L$ la longueur du corps $1, M_{2}$ la masse du corps 2, $Z Z_{1}$ et $Z Z_{2}$ les moments d'inertie des corps 1 et 2 respectivement, $L M X_{2}$ et $L M Y_{2}$ 

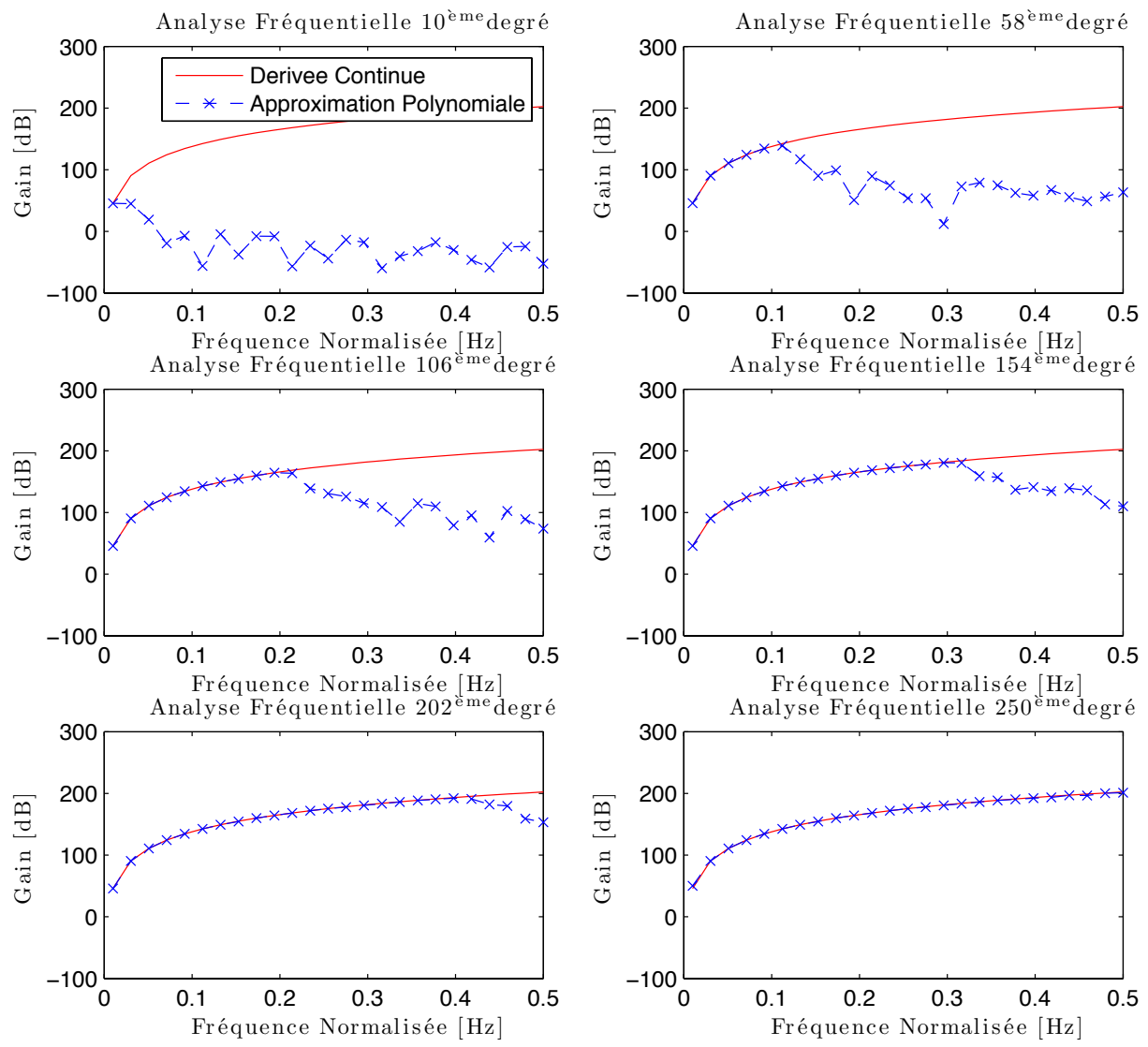

Figure 4. Caractérisation fréquentielle de l'opérateur dérivé d'ordre 2 par projection polynomiale. Fréquence d'échantillonnage de $100 \mathrm{~Hz}$

les moments principaux d'inertie du corps 2 multipliés par $L$, et $F_{v 1}, F_{s 1}, F_{v 2}, F_{s 2}$ respectivement les paramètres de frottements visqueux et secs des articulations 1 et 2 .

\subsection{Méthode d'identification}

Le principe de l'identification consiste à échantillonner le modèle dynamique inverse le long de mouvements excitants en boucle fermée, à différents instants $t_{i}, i \in\left\{1, \ldots, n_{e}\right\}$, de façon à obtenir un système linéaire surdéterminé. Après 


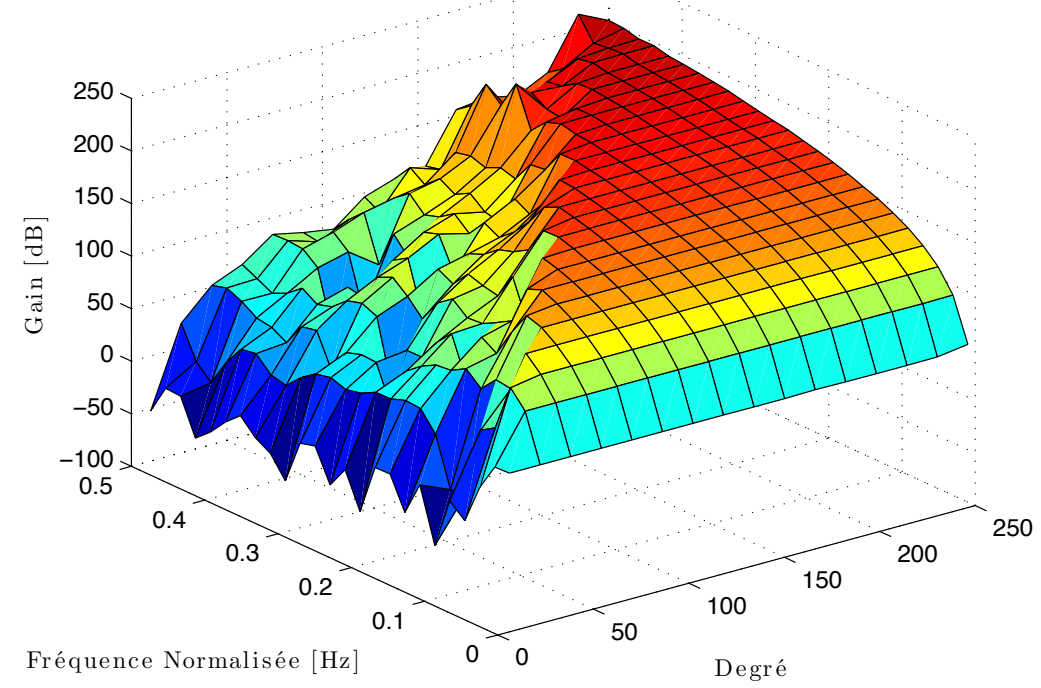

Figure 5. Distorsion en amplitude du filtre dérivateur d'ordre 2 en fonction de la fréquence du signal d'entrée et du degré des polynômes

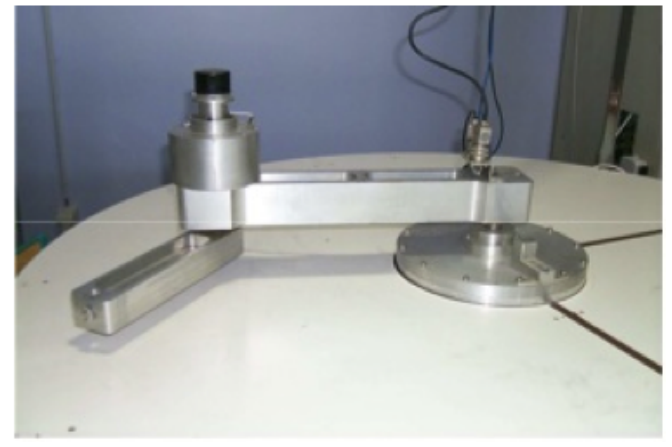

Figure 6. Robot SCARA

échantillonnage de l'équation [17], la concaténation des différentes mesures conduit à l'équation :

$$
Y=W(\widehat{q}, \widehat{\dot{q}}, \widehat{\ddot{q}}) \theta_{S}+\rho
$$

où : 
- $\widehat{q}$, $\widehat{\dot{q}}$ et $\widehat{\ddot{q}}$ sont respectivement les mesures de positions et les estimations des vitesses et accélérations articulaires,

$-Y$ est le vecteur de dimension $\left(2 n_{e} \times 1\right)$ correspondant à l'échantillonnage de l'effort moteur $\Gamma$ du modèle [17],

$-W$ est la matrice de dimension $\left(2 n_{e} \times 8\right)$ correspondant à l'échantillonnage de la matrice $D_{s}$ du modèle [17],

- $\rho$ est le vecteur des résidus dus aux bruits de mesures et aux erreurs de modèle.

À partir de l'équation [20], il est possible d'obtenir une estimation au sens des moindres carrés, notée $\hat{\theta}_{S}$, du vecteur inconnu $\theta_{S}$ :

$$
\hat{\theta}_{S}=\arg \min _{\theta_{S}}\|\rho\|^{2}=W^{+} Y
$$

$W^{+}$désigne la matrice pseudo-inverse de $W$ :

$$
W^{+}=\left(W^{T} W\right)^{-1} W^{T}
$$

Lors de la mise en ouvre pratique, les mesures expérimentales ou les estimations de $\Gamma, \widehat{q}, \widehat{\dot{q}}$ et $\widehat{\ddot{q}}$ sont bruitées. Il en résulte que les matrices $Y$ et $W$ sont perturbées, et que l'estimation des moindres carrés peut présenter un biais si ces matrices aléatoires ne sont pas indépendantes. Les coefficients du régresseur $D_{S}$ [18] sont des fonctions non linéaires de $\widehat{q}, \widehat{\dot{q}}, \widehat{\ddot{q}}$ et il est pratiquement impossible de calculer l'effet théorique de ces perturbations sur le biais et la variance de l'estimation. On adopte donc une stratégie qui vise à minimiser leurs effets en agissant sur le filtrage des données et le choix d'un mouvement excitant optimal. L'unicité de la solution [21] dépend du rang de la matrice d'observation et la perte de rang peut avoir deux origines :

- Une perte de rang structurelle de $W$ peut apparaitre quels que soient les échantillons $(q, \dot{q}, \ddot{q})$ de [20]. Cette perte de rang survient lorsque le modèle d'identification a été paramétré de façon surabondante. Ce problème d'identifiabilité est résolu en utilisant les paramètres de base qui fournissent une représentation minimale du modèle (Mayeda et al., 1990; Gautier and Khalil, 1992).

- Une perte de rang numérique peut être liée à un mauvais choix des échantillons $(q, \dot{q}, \ddot{q})$ sur une trajectoire d'excitation pauvre en informations. Ce problème peut être résolu en réalisant une identification en boucle fermée de position avec suivi de mouvements excitants pour les paramètres (Swevers et al., 1997; Presse and Gautier, 1993). Les mouvements des robots industriels sont définis par des mouvements articulaires point à point (vitesses nulles aux points de passage), avec une interpolation polynomiale entre les points de passage. Le principe consiste à calculer par optimisation non linéaire les coefficients des polynômes qui minimisent une fonction de coût, sous contraintes des positions, vitesses et accélérations admissibles. L'analyse du conditionnement de $W$ montre que celui-ci doit être proche de 1 avec des valeurs singulières grandes, avant de calculer $X_{S}$. De ce constat, (Presse, 1994) suggère la minimisation du critère d'excitation :

$$
f(W)=\operatorname{Cond}(W)+\frac{1}{\Sigma_{p}(W)}=\frac{1+\Sigma_{1}(W)}{\Sigma_{p}(W)}
$$


$\Sigma_{p}$ et $\Sigma_{1}$ désigne respectivement la plus petite et plus grande valeur singulière de $W$. Ce critère tend à uniformiser les estimations des écarts-types des paramètres.

Les matrices $W$ et $Y$ de [20] sont perturbées par les mesures bruitées ou les estimations de $(\widehat{q}, \widehat{\dot{q}}, \widehat{\ddot{q}})$. Il importe donc de filtrer les données avant de calculer la solution au sens des moindres carrés. D'un point de vue pratique, les estimations des dérivées successives de [20] sont obtenues en appliquant la méthode de filtrage présentée dans la section 2.3. Le problème est de bien choisir le paramètre de synthèse $n$ pour que $W(\hat{q}, \hat{\dot{q}}, \hat{\ddot{q}})$ tende vers $W(q, \dot{q}, \ddot{q})$ dans la bande de fréquence du modèle dynamique à identifier. Une distorsion à ce niveau du filtrage introduirait un biais dans l'estimation du vecteur des inconnues $\theta_{S}$. Il a été montré dans (Janot et al., 2009a) que si le filtrage des données est bien conçu, alors la solution au sens des moindres carrés est aussi consistante que celle fournie par une méthode robuste vis-à-vis du bruit présent dans la matrice d'observation telle que la méthode de la variable instrumentale.

\subsection{Résultats expérimentaux}

La fréquence d'échantillonnage pour l'acquisition des données est égale à $100 \mathrm{~Hz}$. Une identification en boucle fermée, utilisant une commande proportionnelle dérivée, a été effectuée avec un suivi de trajectoires excitantes en position constituées de polynômes d'ordre 5. Cette trajectoire a été au préalable calculée par optimisation de manière à optimiser le conditionnement de la matrice d'observation $W$ (Presse and Gautier, 1993; Presse, 1994; Walter and Pronzato, 1997).

Les résultats de l'identification expérimentale sont rapportés dans le tableau 1 avec une base de polynômes de Tchebychev de dimension 125 (estimateur $\hat{\theta}_{S}^{\text {poly }}$ ), et comparés avec :

- une méthode "classique" ( $\left.\hat{\theta}_{S}^{\mathrm{WLS}}\right)$ (Gautier and Poignet, 2001),

- un filtre de Kalman étendu ( $\hat{\theta}_{S}^{\mathrm{EKF}}$ ) (Gautier and Poignet, 2001),

- une méthode de variable instrumentale $\left(\hat{\theta}_{S}^{\mathrm{IV}}\right)$ (Janot et al., 2009a),

- un estimateur de Huber ( $\hat{\theta}_{S}^{\text {Huber }}$ ) (Janot et al., 2009b),

- une technique de dérivation robuste par modes glissants $\left(\hat{\theta}_{S}^{\mathrm{DR}}\right)$ (Sidhom et al., 2010).

Les paramètres estimés sont donnés avec leur écart type relatif. Un paramètre avec un écart type tel que $\% \sigma_{\hat{\theta} r} \geq 10 \%$ signifie qu'il n'est pas ou peu identifiable sur la trajectoire donnée. L'écart-type $\sigma_{\hat{\theta}_{i}}$ sur chaque paramètre estimé est calculé en supposant $\rho$ comme étant un bruit blanc d'écart-type $\sigma_{\rho}$ et de matrice de variance $C_{\rho \rho}$ :

$$
C_{\rho \rho}=\sigma_{\rho}^{2} I_{2 n_{e} \times 2 n_{e}}
$$

où $I_{2 n_{e} \times 2 n_{e}}$ est la matrice identité $\left(2 n_{e} \times 2 n_{e}\right)$. La matrice de variance-covariance de l'erreur d'estimation est donnée par :

$$
C_{\hat{\theta}}=\sigma_{\rho}^{2}\left[W^{T} W\right]^{-1}
$$


$\sigma_{\hat{\theta}_{i}}^{2}=C_{\hat{\theta} i i}$, est le i ìme coefficient de la diagonale de $C_{\hat{\theta}}$. L'écart-type relatif $\% \sigma_{\hat{\theta} r}$ est défini par la relation :

$$
\% \sigma_{\hat{\theta}_{r i}}=100 \frac{\sigma_{\hat{\theta}_{i}}}{\hat{\theta}_{S i}}
$$

Tableau 1. Résultats d'identification

\begin{tabular}{ccccccccc}
\hline $\begin{array}{c}\text { Paramètres } \\
\text { (unités SI) }\end{array}$ & $Z Z R_{1}$ & $F_{v 1}$ & $F_{s 1}$ & $Z Z_{2}$ & $L M X_{2}$ & $L M Y_{2}$ & $F_{v 2}$ & $F_{s 2}$ \\
\hline$\hat{\theta}_{S}^{\text {poly }}$ & 3.43 & 0.04 & 0.75 & 0.061 & 0.123 & 0.008 & 0.033 & 0.098 \\
$\% \sigma_{\hat{\theta}_{r}}$ & 0.2 & 3.6 & 3.0 & 3.6 & 1.6 & 1.6 & 41 & 2.2 \\
\hline$\hat{\theta}_{S}^{\mathrm{WLS}}$ & 3.47 & 0.3 & 0.4 & 0.064 & 0.125 & 0.003 & 0.014 & 0.13 \\
$\% \sigma_{\hat{\theta}_{r}}$ & 0.8 & 25 & 9 & 0.45 & 1.2 & 49 & 6 & 1.7 \\
\hline$\hat{\theta}_{S}^{\mathrm{EKF}}$ & 3.31 & 0.6 & 0.58 & 0.061 & 0.14 & 0.03 & 0.012 & 0.13 \\
$\% \sigma_{\hat{\theta}_{r}}$ & 0.2 & 3.6 & 1.1 & 0.1 & 0.4 & 1.0 & 1.1 & 0.3 \\
\hline$\hat{\theta}_{S}^{\mathrm{IV}}$ & 3.45 & 0.02 & 0.82 & 0.061 & 0.125 & 0.014 & 0.01 & 0.135 \\
$\% \sigma_{\hat{\theta}_{r}}$ & 0.5 & 25 & 5 & 0.4 & 5 & 0.4 & 30 & 7 \\
\hline$\hat{\theta}_{S}^{\text {Huber }}$ & 3.45 & 0.01 & 0.83 & 0.061 & 0.125 & 0.012 & 0.0 & 0.135 \\
$\% \sigma_{\hat{\theta}_{r}}$ & 0.5 & 30 & 4.8 & 0.5 & 0.4 & 5.5 & 100 & 7 \\
\hline$\hat{\theta}_{S}^{\mathrm{DR}}$ & 3.35 & 0.02 & 0.48 & 0.059 & 0.129 & 0.004 & 0.038 & 0.082 \\
$\% \sigma_{\hat{\theta}_{r}}$ & 0.2 & 8.9 & 6.6 & 0.5 & 0.5 & 9.7 & 4.6 & 3.3 \\
\hline
\end{tabular}

Avant tout, on observe que les valeurs et les écarts types de certains paramètres identifiés sont sensiblement les mêmes quelle que soit la méthode utilisée. Il s'agit notamment des paramètres inertiels $Z Z R_{1}$ et $L M X_{2}$. Aucune tendance significative ne différencie particulièrement une méthode par rapport à une autre. Ceci vient principalement du fait que le modèle dynamique inverse est bien spécifié pour notre application et que le filtrage des données est bien conçu. Par suite, les méthodes d'identification sont pratiquement aussi consistantes les unes que les autres. Néanmoins, on remarque que les paramètres de frottements sont toujours assez difficiles à identifier. D'une manière générale, lorsque les paramètres de synthèse peuvent se traduire sous la forme d'un filtrage $\left(\hat{\theta}_{S}^{\text {poly }}, \hat{\theta}_{S}^{\text {WLS }}, \hat{\theta}_{S}^{\text {Huber }}, \hat{\theta}_{S}^{\text {DR }}\right)$, il s'agit de faire coïncider la fréquence de coupure du filtre associé à la méthode avec la bande passante estimée du système. Pour l'observateur de Kalman étendu, on peut montrer que le résultat de l'identification dépend fortement de valeurs initiales et une bonne connaissance des valeurs a priori est conseillée (Gautier and Poignet, 2001). Pour la méthode de la variable instrumentale, il a été montré qu'il n'était pas nécessaire de filtrer les signaux pour obtenir une estimation consistante (Janot et al., 2009a). C'est essentiellement pour cette raison que la méthode est séduisante. Malheureusement, pour appliquer cette méthode, il est pour le moment nécessaire de connaître la structure de la loi de commande utilisée pour contrôler le système. Ceci peut constituer un frein à l'utilisation de la méthode de la variable instrumentale. En effet, les industriels ne délivrent pas toujours cette 
information. S'agissant de la mise œuvre numérique de chaque méthode, les techniques ont été toutes implantées sur Matlab-Simulink. Il ressort que les techniques de filtrage sont peu gourmandes en terme de temps de calculs par rapport au filtre de Kalman (Gautier and Poignet, 2001) et à la méthode de la variable instrumentale (Janot et al., 2009a) qui nécessitent la simulation et l'intégration du modèle dynamique direct. Notons que certaines techniques comme le différentiateur robuste à base de modes glissants (Sidhom et al., 2010) permettent d'envisager une identification en ligne. À présent, la complexité algorithmique de chaque méthode dépend essentiellement de l'aisance du programmeur avec la technique considérée. Pour finir, afin de valider définitivement cette identification, nous avons effectué des comparaisons directes : les couples de commande sont reconstitués à partir des paramètres identifiés et sont comparés avec les couples mesurés. Les résultats sont illustrés sur la figure 7. Le couple estimé suit de manière fidèle celui mesuré, ceci témoigne de la bonne qualité de l'identification.

a) Validation Directe : Axe 1

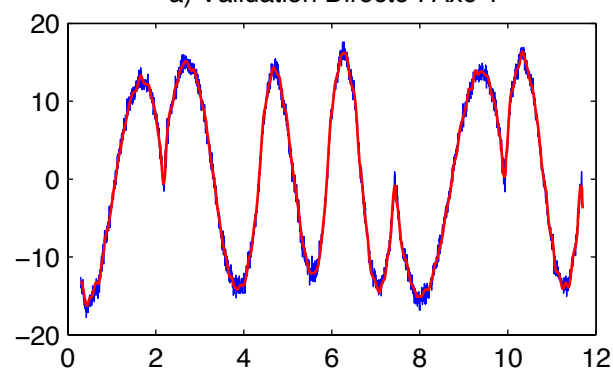

c) Validation Directe : Axe 1

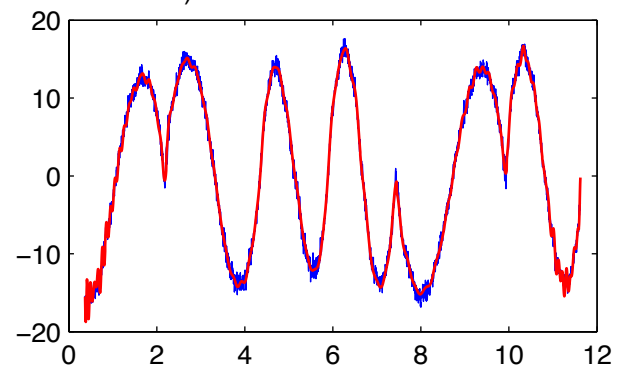

b) Validation Directe : Axe 2

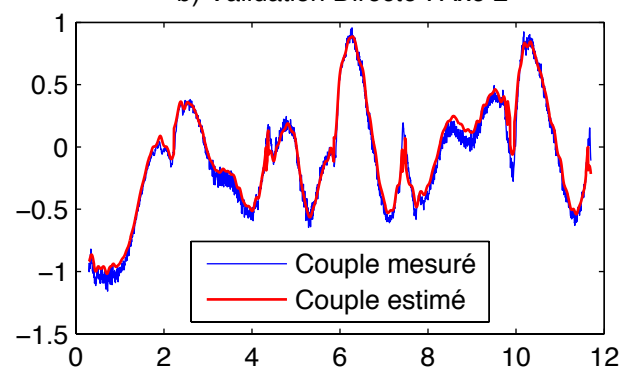

d) Validation Directe : Axe 2

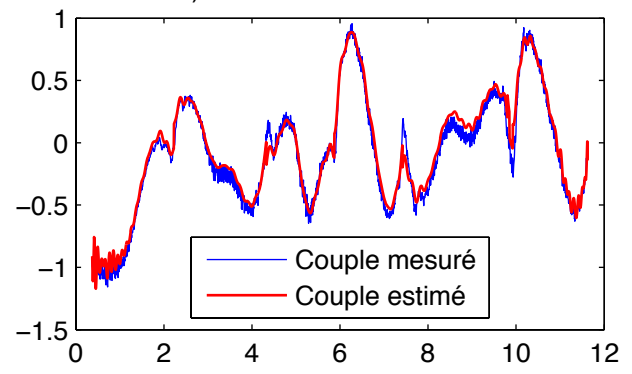

Figure 7. Validation directe des efforts mesurés (en bleu) et simulés (en rouge) : a) et b) par une méthode de moindres carrés. c) et d) par la méthode de projection polynomiale 


\section{Conclusion}

Dans ce article, une présentation des propriétés des polynômes de Tchebychev et une formulation dérivée utilisant ces polynômes ont été effectuées. Comme tout problème d'identification à temps continu, l'hypothèse de la présence d'un bloqueur d'ordre zéro sur l'entrée du système n'est pas nécessaire. La méthode d'identification présentée consiste à appliquer une transformation linéaire sur le système d'équations qui régit le processus. Pour cela, l'opérateur mathématique utilisé s'appuie sur une décomposition du signal dans une base formée de polynômes orthogonaux. Nous avons montré que cette projection se comporte comme un filtre passe-bande, de telle sorte qu'une seule opération est nécessaire pour le pré-traitement des données avant le processus d'identification à savoir la projection des signaux sur la base de fonctions de Tchebychev, régie par la dimension de cette base. La méthodologie développée est expérimentée dans le cadre difficile de l'identification dynamique des robots. En raison du caractère double intégrateur du système considéré, l'identification expérimentale est menée en boucle fermée. De ce fait, il en résulte que la matrice d'observation est bruitée. D'un point de vue théorique, l'estimateur des moindres carrés est biaisé si cette matrice et le vecteur contenant les couples articulaires ne sont pas indépendants. Pour minimiser l'effet des perturbations sur l'estimation la stratégie adoptée consiste d'une part à utiliser un filtrage approprié des données pour obtenir un modèle d'identification filtré, peu bruité. D'autre part, les mouvements utilisés en boucle fermée sont suffisamment riche en informations pour exciter les paramètres. Dans la pratique des perturbations comme le bruit de quantification lié au capteur de position n'a quasiment pas d'influence et, par conséquent, l'estimateur des moindres carrés n'est pratiquement pas biaisé. Une identification expérimentale en boucle fermée d'un robot à 2 axes avec cette méthode est effectuée dans une dernière partie. Les résultats fournis par notre méthode ont été comparés avec ceux obtenus avec différentes techniques. Comme les résultats sont tous assez proches les uns des autres, notre démarche proposée est parfaitement valable.

\section{Annexes}

\subsection{Expression des coefficients $\tilde{x}_{i}$}

Considérons l'échantillonnage de la fonction $x: t \mapsto x(t)$ aux instants $t_{i} \in$ $\left\{t_{0}, \ldots, t_{f}\right\}$. On suppose que la fonction $x$ est linéaire par morceaux :

$$
\forall i \in\{0, \ldots, f-1\}, \quad x(t)=x\left(t_{i}\right)+\frac{x\left(t_{i+1}\right)-x\left(t_{i}\right)}{t_{i+1}-t_{i}}\left(t-t_{i}\right) \quad \operatorname{sur}\left[t_{i}, t_{i+1}\right]
$$


Les coefficients $\tilde{x}_{i}$ du développement [5] de $x$ sur la base de Tchebychev sont donnés par [9], avec :

$$
\begin{aligned}
<x_{I}, P_{i}> & =\int_{-1}^{1} \frac{x_{I}(\tau) \cos (i \arccos (\tau))}{\sqrt{1-\tau^{2}}} d \tau \\
& =\sum_{j=0}^{f-1}\left(x\left(t_{j}\right)-\frac{x\left(t_{j+1}\right)-x\left(t_{j}\right)}{\tau_{j+1}-\tau_{j}} \tau_{j}\right) I_{i j}+\sum_{j=0}^{f-1} \frac{x\left(t_{j+1}\right)-x\left(t_{j}\right)}{\tau_{j+1}-\tau_{j}} J_{i j}
\end{aligned}
$$

où $\tau_{j}=\tau\left(t_{j}\right)$,

$$
\begin{aligned}
I_{i j} & =\int_{\tau_{j}}^{\tau_{j+1}} \frac{\cos (i \arccos (\tau))}{\sqrt{1-\tau^{2}}} d \tau \\
& = \begin{cases}{[\arcsin (\tau)]_{\tau_{j}}^{\tau_{j+1}}} & \text { si } i=0 \\
-\frac{1}{i}[\sin (i \arccos (\tau))]_{\tau_{j}}^{\tau_{j+1}} & \text { si } i \geq 1\end{cases}
\end{aligned}
$$

et

$$
\begin{aligned}
J_{i j} & =\int_{\tau_{j}}^{\tau_{j+1}} \frac{\tau \cos (i \arccos (\tau))}{\sqrt{1-\tau^{2}}} d \tau \\
& =\left\{\begin{array}{cc}
-[\sin (\arccos (\tau))]_{\tau_{j}}^{\tau_{j+1}} & \text { si } i=0 \\
-\frac{1}{4}[2 \arccos (\tau)+\sin (2 \arccos (\tau))]_{\tau_{j}}^{\tau_{j+1}} & \text { si } i=1 \\
\frac{1}{i^{2}-1}[\sin (\arccos (\tau)) \cos (i \arccos (\tau)) & \\
-i \tau \sin (\arccos (\tau))]_{\tau_{j}}^{\tau_{j+1}} & \text { si } i \geq 2
\end{array}\right.
\end{aligned}
$$

\subsection{Expression de la matrice de dérivation}

À titre d'information, la matrice $D$ dans [13] est donnée par [27], dans le cas de Tchebychev type I, pour $n$ pair (Rémond et al., 2008; Lizandier et al., 2011) :

$$
D=\left(\begin{array}{ccccccccc}
0 & 0 & 0 & 0 & 0 & 0 & \ldots & 0 & 0 \\
1 & 0 & 0 & 0 & 0 & 0 & \ldots & 0 & 0 \\
0 & 4 & 0 & 0 & 0 & 0 & \ldots & 0 & 0 \\
3 & 0 & 6 & 0 & 0 & 0 & \ldots & 0 & 0 \\
0 & 8 & 0 & 8 & 0 & 0 & \ldots & 0 & 0 \\
5 & 0 & 10 & 0 & 10 & 0 & \ldots & 0 & 0 \\
\vdots & \vdots & \vdots & \vdots & \vdots & \vdots & \ddots & \vdots & \vdots \\
n-1 & 0 & 2(n-1) & 0 & 2(n-1) & 0 & \ddots & 0 & 0 \\
0 & 2 n & 0 & 2 n & 0 & 2 n & \ldots & 2 n & 0
\end{array}\right)
$$


Dans le cas où $n$ est impair (Rouby et al., 2010), il vient :

$$
D=\left(\begin{array}{ccccccccc}
0 & 0 & 0 & 0 & 0 & 0 & \ldots & 0 & 0 \\
1 & 0 & 0 & 0 & 0 & 0 & \ldots & 0 & 0 \\
0 & 4 & 0 & 0 & 0 & 0 & \ldots & 0 & 0 \\
3 & 0 & 6 & 0 & 0 & 0 & \ldots & 0 & 0 \\
0 & 8 & 0 & 8 & 0 & 0 & \ldots & 0 & 0 \\
5 & 0 & 10 & 0 & 10 & 0 & \ldots & 0 & 0 \\
\vdots & \vdots & \vdots & \vdots & \vdots & \vdots & \ddots & \vdots & \vdots \\
0 & 2(n-1) & 0 & 2(n-1) & 0 & 2(n-1) & \ldots & 0 & 0 \\
n & 0 & 2 n & 0 & 2 n & 0 & \ddots & 2 n & 0
\end{array}\right)
$$

\section{Bibliographie}

Ahmed S., Huang B., Shah S., « Parameter and delay estimation of continuous-time models using a linear filter », Journal of Process Control, vol. 16, n 4, p. 323-331, 2006.

Chen Q., Tomlinson G., «Parametric identification of systems with dry friction and nonlinear stiffness using a time series model », Transactions of ASME Journal of Vibrations and Acoustics, vol. 118, n 2, p. 252-263, 1996.

Chou C., Verhaegen M., Johansson R., «Continuous-time identification of siso systems using Laguerre functions », IEEE Transactions on Signal Processing, vol. 47, n 2, p. 349-362, 1999.

Gautier M., Contribution à la modélisation et à l'identification des robots, Thèse de doctorat d'état, Université de Nantes, École Nationale Supérieure de la Mécanique, 1990.

Gautier M., Khalil W., « Direct calculation of the base inertial parameters », IEEE Transactions on Robotics and Automation, vol. 6, n 3, p. 368-373, 1990.

Gautier M., Khalil W., « Exciting trajectories for the identification of base inertial parameters of robots », International Journal of Robotic Research, vol. 1, p. 362-375, 1992.

Gautier M., Poignet P., « Extended Kalman filtering and weighted least squares dynamic identification of robot », Control Engineering Practice, vol. 9, p. 1361-1372, 2001.

Ghanem R., Romeo F., «A wavelet-based approach for the identification of linear time varying systems », Journal of Sound and Vibration, vol. 234, $\mathrm{n}^{\circ}$ 4, p. 555-576, 2000.

Ghanem R., Romeo F., «A wavelet-based approach for model and parameter identification of non-linear systems », International Journal of Non-Linear Mechanics, vol. 36, n 5, p. 835859, 2001.

Horng I., Chou J., « Analysis and identification of nonlinear systems via shifted Jacobi series », International Journal of Control, vol. 45, $\mathrm{n}^{\circ}$ 1, p. 279-290, 1987.

Horng I. R., Chou J. H., « Analysis and parameter identification of time-delay systems via shifted Jacobi polynomials », International Journal of Control, vol. 44, p. 935-942, 1986.

Janot A., Vandanjon P. O., Gautier M., «Identification of Robot Dynamics with the Instrumental Variable Method », IEEE International Conference on Robotics and Automation (ICRA), Kobe, Japon, p. 1762-1767, 2009a. 
Janot A., Vandanjon P. O., Gautier M., «Using robust regressions and residual analysis to verify the reliability of LS estimation: Application in robotics », IEEE International Conference on Intelligent Robots and Systems (IROS), Saint Louis, USA, p. 1962-1967, 2009b.

Karanam V., Frick P., Mohler R., «Bilinear system identification by Walsh functions », IEEE Transactions on Automatic Control, vol. 23, n 4, p. 709-713, 1978.

Khalil W., Dombre E., Modeling, Identification and Control of Robots, Elsevier Butterworth Heinemann, 1999.

Kozlowski K., Modelling and identification in robotics, Springer, London, 1998.

Larsson E., Soderstrom T., «Identification of continuous-time AR processes from unevenly sampled data », Automatica, vol. 38, n 4, p. 709-718, 2002.

Lizandier J., Pham M. T., Rémond D., Rouby C., «Utilisation de Polynômes de Chebychev pour l'Identification à Temps Continu », Journées Identification et Modélisation Expérimentale, Douai, April 6-7, 2011.

Masri S., Caughey T., «A nonparametric identification technique for nonlinear dynamic problems », Transactions of ASME Journal of Applied Mechanics, vol. 46, p. 433-447, 1979.

Mayeda H., Yoshida K., Osuka K., «Base parameters of manipulator dynamic models », IEEE Transactions On Robotics and Automation, vol. 6, n 3, p. 312-321, 1990.

Mensler M., Analyse et étude comparative de méthodes d'identification des systèmes à représentation continue, Développement d'une boîte à outils logicielle, PhD thesis, UFR Sciences et Techniques Université Poincaré, 1999.

Mohan B. M., Datta K. B., «Analysis of time-delay systems via shifted Chebyshev polynomials of the first and second kinds ", International Journal of Systems Science, vol. 19, $\mathrm{n}^{\circ}$ 9, p. 1843-1851, 1988.

Mohan B. M., Datta K. B., «Identification via Fourier series for a class of lumped and distributed parameter systems », IEEE Transactions on Circuits and Systems, vol. 36, $\mathrm{n}^{\circ} 11$, p. 1454-1458, November, 1989.

Pacheco R., Steffen V., «Using orthogonal functions for identification and sensitivity analysis of mechanical systems », Journal of Vibration and Control, vol. 8, n 7, p. 993-1021, 2002.

Pacheco R., Steffen V., « On the identification of non-linear mechanical systems using orthogonal functions », International Journal of Non-Linear Mechanics, vol. 39, n 7, p. 1147-1159, 2004.

Paraskevopoulos P. N., Kekkeri G., «Hermite series approach to system identification, analysis and optimal control », Proceedings Measurement and Control, vol. 2, p. 146-149, 1983.

Paraskevopoulos P. N., Mouroutsos S. G., «Legendre series approach to identification and analysis of linear systems », IEEE Transactions Automatic Control, vol. 30, p. 585-589, 1985.

Presse C., Identification des paramètres dynamiques des robots, Thèse de doctorat, Université de Nantes-École Centrale de Nantes, 1994.

Presse C., Gautier M., « New criteria of exciting trajectories for robot identification », IEEE international conference on robotics and automation, Atlanta, Georgia, USA, p. 907-912, 1993.

Rémond D., Neyrand J., Aridon G., Dufour R., « On the improved use of Chebyshev expansion for mechanical system identification », Mechanical Systems and Signal Processing, vol. 22, p. 390-407, 2008. 
Rouby C., Rémond D., Argoul P., « Orthogonal polynomials or wavelet analysis for mechanical system direct identification », Annals of Solid and Structural Mechanics, vol. 1, n 1, p. 4158, December, 2010.

Sciavicco L., Siciliano B., Modelling and Control of Robot Manipulators, Mac Graw-Hill, 2000.

Sidhom L., Pham M. T., Thevenoux F., Gautier M., «Identification of a robot manipulator based on an adaptive higher order sliding modes differentiator », IEEE/ASME International Advanced Intelligent Mechatronics (AIM), Montreal, Canada, p. 1093-1098, July 6-9, 2010.

Swevers J., Ganseman C., Tükel D. B., de Schutter J., van Brussel H., «Optimal robot excitation and identification », IEEE Transactions on Robotics and Automation, vol. 13, n 5, p. 730740, 1997.

Walter E., Pronzato L., Identification of Parametric Models from Experimental Data, Springer, 1997.

Young P., « Parameter estimation for continuous-time models-a survey. », Automatica, vol. 17, $\mathrm{n}^{\circ} 1$, p. 23-39, 1981. 



\section{ANNEXE POUR LE SERVICE FABRICATION A FOURNIR PAR LES AUTEURS AVEC UN EXEMPLAIRE PAPIER DE LEUR ARTICLE ET LE COPYRIGHT SIGNE PAR COURRIER LE FICHIER PDF CORRESPONDANT SERA ENVOYE PAR E-MAIL}

1. ARTIClE POUR LA REVUE :

JESA

\section{Auteurs :}

Minh Tu PHAM ${ }^{1}$ - Corinne Roub ${ }^{2}$ - Jérémy LIZANDIER 1 - Didier RÉMOND $^{3}$ - Alexandre JANOT ${ }^{4}$ - Pierre-Olivier VANDANJON ${ }^{5}-$ Maxime GAUTIER 6

\section{TITRE DE L'ARTICLE :}

Utilisation de Polynômes de Tchebychev pour l'Identification de Modèles à Temps Continu de Robots

4. TITRE ABRÉGÉ POUR LE HAUT DE PAGE MOINS DE 40 SIGNES :

Identification Polynômiale

\section{DATE DE CETTE VERSiON :}

22 février 2012

6. CoOrdonnéEs des Auteurs :

- adresse postale :

1 Laboratoire Ampère, UMR CNRS 5005,

Université de Lyon, INSA-Lyon, F-69621 Villeurbanne Cedex, France

(minh-tu.pham, jeremy.lizandier)@insa-lyon.fr

2 Unité de Mécanique,

ENSTA ParisTech, Chemin de le Hunière, 91761 Palaiseau Cedex, France corinne.rouby@ensta-paristech.fr

3 LaMCoS, CNRS UMR5259, Université de Lyon, INSA-Lyon, F-69621 Villeurbanne Cedex, France didier.remond@insa-lyon.fr

4 ONERA DCSD,

2 Avenue Edouard Belin - BP 74025, 31055 Toulouse Cedex 04, France

Alexandre.Janot@onera.fr

5 Laboratoire Central des Ponts et Chaussées, 44341 Bouguenais Cedex, France pierre-olivier.vandanjon@ @ lcpc.fr

6 IRCCyN, UMR CNRS 6597, Université de Nantes, 44321 Nantes Cedex 03, France maxime.gautier@irccyn.ec-nantes.fr

- téléphone : 0000000000 
SERVICE ÉDITORIAL - HERMES-LAVOISIER 14 rue de Provigny, F-94236 Cachan cedex

Tél. : 01-47-40-67-67

E-mail : revues@lavoisier.fr

Serveur web : http://www.revuesonline.com 